\title{
IUFOST2006/1090 \\ Liking, wanting, and eating: Too much of a good thing?
}

\author{
D. Mela \\ Unilever Food \& Health Research Institute, Olivier van Noortlaan 120, PO Box 114, 3130 AC Vlaardingen, \\ Netherlands \\ david.mela@unilever.com
}

\begin{abstract}
Consumers demand foods that are attractive and pleasurable to eat, but sensory stimulation can influence energy intake in the short-term, leading to speculation about food "palatability" as a potential contributor to longer-term overeating and obesity. However, while the "tastes better -> eat more" notion seems obvious, the evidence, nature and dynamics of this relationship are not. Small-scale laboratory experiments and surveys probably exaggerate the size and duration of the effects of sensory influences on intake, and often mix effects of food sensory quality with effects of food composition. In contrast, real-world variation in diet "palatability" may explain relatively little of variance in energy intakes. Furthermore, obesity is not reliably associated with a heightened liking of foods in general.

Several underlying processes need to be considered and distinguished, in order to make sense of the palatabilityintake relationship in relation to obesity. These include: - Relative influence and co-variance of food sensory quality vs other influences on selection and intake - Independent effects of food variety, composition, and accessibility - Acquisition of liking for energy-rich foods - Nature of "wanting" (motivation to eat) vs "liking" (immediate pleasure)

A more advanced view of the palatability-intake relationship has important implications for further research, and its application in commercial and public health approaches to modifying energy intakes.
\end{abstract}

